\title{
NEW CHARACTERIZATION OF APPELL POLYNOMIALS
}

\author{
ABDELMEJID BAYAD AND TAKAO KOMATSU*
}

\begin{abstract}
We prove characterizations of Appell polynomials by means of symmetric property. For these polynomials, we establish a simple linear expression in terms of Bernoulli and Euler polynomials. As applications, we give interesting examples. In addition, from our study, we obtain Fourier expansions of Appell polynomials. This result recovers Fourier expansions known for Bernoulli and Euler polynomials and obtains the Fourier expansions for higher order BernoulliEuler's one.
\end{abstract}

\section{INTRODUCTION}

We give a definition as broad as possible for the so-called sequences of Appell polynomials. This definition is based on the Appell's original work [1] published in 1882.

Let us fix $g, \varphi$ two functions. The function $g: \mathbb{C} \rightarrow \mathbb{C}$ is holomorphic at $0, g(0)=0, g^{\prime}(0) \neq 0$, and $\varphi: \mathbb{N} \rightarrow \mathbb{C} \backslash\{0\}$ an arbitrary function.

Let $\left(P_{n}(x)\right)_{n}$ be a sequence of polynomials. We call $\left(P_{n}(x)\right)_{n}$ a sequence of Appell polynomials of type $(g, \varphi)$, if and only if there exists $f: \mathbb{C} \rightarrow \mathbb{C}$ holomorphic at 0 such that $f(0) \neq 0$ and

$$
\sum_{n \geq 0} P_{n}(x) \frac{t^{n}}{\varphi(0) \cdots \varphi(n)}=f(t) e^{x g(t)} .
$$

The ordinary Appell sequence of polynomials corresponds to special type

$$
g(t)=t, \varphi(0)=1, \text { and } \varphi(n)=n, n \geq 1 .
$$

In addition, for the above type we have Bernoulli's polynomials $B_{n}(x)$ corresponds to $f_{B}(t)=\frac{t}{e^{t}-1}$, and Euler's polynomials corresponds to $f_{E}(t)=\frac{2}{e^{t}+1}$.

However for this paper, without loss of generality, we can assume that $\varphi(n)=n$ for $n \geq 1$ and $\varphi(0)=1$. To see this we put

and rewrite the relation (1.1)

$$
Q_{n}(x)=P_{n}(x) \frac{n !}{\varphi(0) \cdots \varphi(n)}
$$

$$
f(t) e^{x g(t)}=\sum_{n \geq 0} P_{n}(x) \frac{t^{n}}{\varphi(0) \cdots \varphi(n)}=\sum_{n \geq 0} Q_{n}(x) \frac{t^{n}}{n !} .
$$

2000 Mathematics Subject Classification. Primary: 33C65 , 39B32; Secondary: 11B68, 42A16.

Key words and phrases. Appell sequences, Generating function, Bernoulli polynomials, Euler polynomials, Fourier expansions.

${ }^{*}$ Corresponding Author. komatsu@whu.edu.cn. 
In this paper, for fixed analytic function $g$ we study the classes of sequence of Appell polynomials $\left(P_{n}(x)\right)_{n}$ in 1.1 when they satisfy the symmetric relation

$$
P_{n}(a-x)=(-1)^{n} P_{n}(x)
$$

for some real parameter $a$.

\subsection{Known Characterizations: An overview.}

We review the known results concerning special cases of ordinary Appell sequences of polynomials. Namely, Bernoulli and Euler polynomials. According to Bernoulli, Euler, Appel, Hurwitz, Raabe, Lucas. There are several approaches to study Bernoulli and Euler polynomials. Here, we list them :

(1) Generating functions theory (Euler [4])

$$
\begin{gathered}
\sum_{n \geq 0} B_{n}(x) \frac{t^{n}}{n !}=\frac{t \cdot e^{x t}}{e^{t}-1},|t|<2 \pi . \\
\sum_{n \geq 0} E_{n}(x) \frac{t^{n}}{n !}=\frac{2 \cdot e^{x t}}{e^{t}+1},|t|<\pi .
\end{gathered}
$$

(2) Appell sequence theory (Appell [1] )

$$
\begin{aligned}
& \frac{d}{d x} B_{n}(x)=n \cdot B_{n-1}(x) \\
& \frac{d}{d x} E_{n}(x)=n \cdot E_{n-1}(x) .
\end{aligned}
$$

(3) Umbral Calculus (Lucas [8])

$$
B_{n}(x)=(B+x)^{n} .
$$

(4) Fourier Series (Hurwitz [5])

$$
\begin{gathered}
B_{n}(x)=\frac{-(n !)}{(2 \pi i)^{n}} \sum_{0 \neq k \in \mathbb{Z}} \frac{e^{2 \pi i k x}}{k^{n}}, 0<x<1 . \\
E_{n}(x)=\frac{2 \cdot(n !)}{(2 \pi i)^{n+1}} \sum_{k \in \mathbb{Z}} \frac{e^{2 \pi i\left(k+\frac{1}{2}\right) x}}{\left(k+\frac{1}{2}\right)^{n+1}}, 0<x<1 .
\end{gathered}
$$

(5) Raabe multiplication theorem [9]

$$
\begin{gathered}
\sum_{k=0}^{m-1} B_{n}\left(\frac{x+k}{m}\right)=m^{1-n} B_{n}(x), \forall m \geq 1, \forall n \in \mathbb{N} . \\
\sum_{k=0}^{m-1}(-1)^{k} E_{n}\left(\frac{x+k}{m}\right)=m^{-n} E_{n}(x), \forall m \geq 1 \text { odd }, \forall n \in \mathbb{N} .
\end{gathered}
$$


We refer to Lehmer's paper [7] for concise details about those approaches. All these Approachs can be generalized to any generalized Appell sequences polynomials. We omit this point and leave the details to the reader.

Our goal, in this paper, we investigate new Approach by means a Symmetry relation. To our knowledge this approach has not yet been exploited.

\section{StATEMENT OF MAIN RESUltS}

For this section we consider fixed type $(g, \varphi)$ Appell sequences of polynomials.

2.1. Characterization of Appell sequences polinomials of type $(g, \varphi)$. We state our first main result.

Theorem 2.1 (First main result). Let a be real parameter. We set

$$
h(t):=f(t) e^{\frac{a}{2} g(t)}
$$

and denote by

$$
V(a):=\left\{\left(P_{k}\right)_{k} \text { Appell polynomials sequence (1.1) } \mid P_{k}(a-x)=(-1)^{k} P_{k}(x)\right\} .
$$

We have

$$
V(a) \neq \emptyset \Longleftrightarrow g \text { is odd, and } h \text { is even. }
$$

Proof. We have $V(a) \neq \emptyset$ if and only if there is $\left(P_{k}\right)_{k}$ sequence of Appell polynomials such that

$$
\sum_{n \geq 0} P_{n}(a-x) \frac{t^{n}}{\varphi(0) \cdots \varphi(n)}=\sum_{n \geq 0}(-1)^{n} P_{n}(x) \frac{t^{n}}{\varphi(0) \cdots \varphi(n)}
$$

The relation (2.1) is equivalent to

$$
f(t) e^{(a-x) g(t)}=f(-t) e^{x g(-t)}, \forall x,
$$

thus, $V(a) \neq \emptyset$ if and only if $h(t)=h(-t) e^{x(g(t)+g(-t))}, \forall x$.

Then $V(a) \neq \emptyset$ if and only if $g$ is odd and $h$ is even.

Corollary 2.2. For $a=0$ we have the function $h(t)=f(t)$. Then $V(0) \neq \emptyset$ if and only if $g$ is odd and $f$ is even.

Theorem 2.3. Let $a \neq 0$ be real parameter. We have the following characterization for the set $V(a)$ of Appell sequences of polynomials. We have $V(a) \neq \emptyset$ if and only if the functions $g$ and $t \rightarrow\left(e^{a g(t)}-1\right) f(t)$ are odd.

Proof. Write $f_{+}, f_{-}$the even and odd part of $f$, respectively. Thanks to Theorem 2.1 we explore the parity of the functions $h$ and $g$. We have

$$
h(t)=\left(f_{+}(t)+f_{-}(t)\right) e^{\frac{a}{2} g(t)}=h(-t)=\left(f_{+}(t)-f_{-}(t)\right) e^{-\frac{a}{2} g(t)},
$$


which is equivalent to $f(t)\left(1-e^{a g(t)}\right)=2 f_{-}(t)$. Then the functions $g$ and $t \rightarrow\left(e^{a g(t)}-1\right) f(t)$ are odd.

Conversely, put $\psi(t)=\left(e^{a g(t)}-1\right) f(t)$ which is odd function. Then we have

$$
\sum_{n \geq 0} P_{n}(a-x)(a-x) \frac{t^{n}}{\varphi(0) \cdots \varphi(n)}=f(t) e^{(a-x) g(t)}
$$

and

$$
\begin{aligned}
& f(t) e^{(a-x) g(t)}=\frac{\psi(t)}{e^{a g(t)}-1} e^{(a-x) g(t)} \\
& =\frac{\psi(-t)}{e^{a g(-t)}-1} e^{x g(-t)} \\
& =f(-t) e^{x g(-t)}
\end{aligned}
$$

hence

$$
P_{k}(a-x)=(-1)^{k} P_{k}(x), \forall k .
$$

Thus we obtain our desired result.

2.2. Application to type $g(t)=t$. For this section we fix the type $g(t)=t$ and $\varphi(0)=1, \varphi(n)=$ $n, n \geq 1$. Next we will describe the set $V(a)$ explicitly, by truncating the Appell sequences (1.1). Denote by

$$
V_{n}(a)=\left\{P \in \mathbb{C}_{n}[x] \mid \exists\left(P_{k}\right)_{k} \in V(a): P=P_{k_{0}} \text { for some } k_{0} \in \mathbb{N}\right\} .
$$

Theorem 2.4 (Second main result). Let $n$ be a positive integer, and $0 \neq$ a real parameter. We have

$$
V_{n}(a)=\operatorname{Vect}\left(B_{n-2 k}(x / a) ; 0 \leq k \leq n / 2\right)
$$

is $V_{n}(a)$ is the subspace spanned by $\left\{B_{n-2 k}(x / a) ; 0 \leq k \leq n / 2\right\}$, with dimension equal to $[n / 2]+1$, alternatively,

$$
V_{n}(a)=\operatorname{Vect}\left(E_{n-2 k}(x / a) ; 0 \leq k \leq n / 2\right) .
$$

By symmetry properties of Bernoulli and Euler polynomials it is easy to see that $V_{n}(a)$ contains $\operatorname{Vect}\left(B_{n-2 k}(x / a) ; 0 \leq k \leq n / 2\right)$ and $\operatorname{Vect}\left(E_{n-2 k}(x / a) ; 0 \leq k \leq n / 2\right)$. To prove the converse we need more preliminaries.

We start to prove the following two theorems.

Theorem 2.5. Let a be a nonzero real parameter, and $\left(P_{n}(x)\right)_{n}$ be a sequence of Appell polynomials of type $(g, \varphi)$ such that

$$
P_{n}(a-x)=(-1)^{n} P_{n}(x) .
$$

Let $\left(a_{k}\right)_{k \in \mathbb{N}}$ be sequence of real numbers such that the function

$$
F: t \rightarrow f(t)-\sum_{k} a_{k} \frac{t^{k}}{k !} \text { is odd or even }
$$


Then we obtain

$$
\begin{aligned}
& P_{n}(x)=\sum_{k \text { even }} a_{k}\left(\begin{array}{l}
n \\
k
\end{array}\right) a^{n-k} E_{n-k}\left(\frac{x}{a}\right), \text { if } F \text { is odd } \\
& P_{n}(x)=-2 \sum_{k \text { odd }} a_{k} \frac{1}{k}\left(\begin{array}{c}
n \\
k-1
\end{array}\right) a^{n-k+1} B_{n-k+1}\left(\frac{x}{a}\right), \text { if } F \text { is even } .
\end{aligned}
$$

Proof. If the relation $P_{n}(a-x)=(-1)^{n} P_{n}(x)$ and (1.1) hold, we obtain

$$
f(t) e^{a t}=f(-t)
$$

and hence we have

$$
F(t) e^{a t}+\sum_{k} a_{k} \frac{t^{k}}{k !} e^{a t}-\sum_{k} a_{k} \frac{(-t)^{k}}{k !}=F(-t) .
$$

If $F$ is odd, then we have

$$
F(t)=\sum_{k} a_{k} \frac{t^{k}}{k !}\left[\frac{(-1)^{k}-e^{a t}}{e^{a t}+1}\right] .
$$

Therefore we obtain

$$
\begin{aligned}
f(t) & =\sum_{k} a_{k} \frac{t^{k}}{k !}\left[\frac{(-1)^{k}+1}{e^{a t}+1}\right], \\
& =\sum_{k \text { even }} a_{k} \frac{t^{k}}{k !} \frac{2}{e^{a t}+1} .
\end{aligned}
$$

Thus

$$
f(t) e^{x t}=\sum_{k \text { even }} a^{-k} a_{k} \frac{(a t)^{k}}{k !} \cdot \frac{2 e^{\frac{x}{a}(a t)}}{e^{a t}+1} .
$$

By use of the equations (1.5) and (1.1), we complete the proof of identity (2.5).

Similarly, from the equations (1.4), (1.1) we prove the identity (2.6) and also get

$$
f(t) e^{x t}=-2 \sum_{k \text { odd }} a^{-k+1} a_{k} \frac{(a t)^{k-1}}{k !} \cdot \frac{(a t) e^{\frac{x}{a}(a t)}}{e^{a t}-1} .
$$

2.3. Fourier expansions for Appell polynomials of type $g(t)=t$. For $0<x<1$ if $n=1,0 \leq$ $x \leq 1$ if $n \geq 2$. It is well-known that

$$
B_{n}(x)=\frac{-n !}{(2 \pi i)^{n}} \sum_{k \in \mathbb{Z} \backslash\{0\}} \frac{e^{2 \pi i k x}}{k^{n}} .
$$

Concerning Euler's polynomials, for $0<x<1$ if $n=0,0 \leq x \leq 1$ if $n \geq 1$. We have

$$
E_{n}(x)=\frac{2(n !)}{(2 \pi i)^{n+1}} \sum_{k \in \mathbb{Z}} \frac{e^{2 \pi i\left(k-\frac{1}{2}\right) x}}{\left(k-\frac{1}{2}\right)^{n+1}} .
$$

(2.8) and (2.9). 
Theorem 2.6. Let a be a nonzero real parameter, and $\left(P_{n}(x)\right)_{n}$ be a sequence of Appell polynomials of type $(g, \varphi)$ such that

$$
P_{n}(a-x)=(-1)^{n} P_{n}(x) .
$$

Let $\left(a_{k}\right)_{k \in \mathbb{N}}$ be sequence of real numbers such that the function

$$
F: t \rightarrow f(t)-\sum_{k} a_{k} \frac{t^{k}}{k !} \text { is odd or even }
$$

(1) For $F$ odd, $0<x / a<1$ if $n=0,0 \leq x / a \leq 1$ if $n \geq 1$, and write

$$
c_{m}^{-}(a):=\sum_{k \text { even }} \frac{a_{k}}{k !}\left(\frac{\pi i}{a}\right)^{k}(2 m-1)^{k} .
$$

Then we have

$$
P_{n}(x)=\frac{2 a^{n}(n !)}{(2 \pi i)^{n+1}} \sum_{m \in \mathbb{Z}} c_{m}^{-}(a) \frac{e^{2 \pi i\left(m-\frac{1}{2}\right) x}}{\left(m-\frac{1}{2}\right)^{n+1}} .
$$

(2) For $F$ even, $0<x / a<1$ if $n=1,0 \leq x / a \leq 1$ if $n \geq 2$, and write

$$
c_{m}^{+}(a):=\sum_{k \text { odd }} \frac{a_{k}}{k !}\left(\frac{\pi i}{a}\right)^{k-1} m^{k-1} .
$$

Then we have

$$
P_{n}(x)=-\frac{2 a^{n}(n !)}{(2 \pi i)^{n+1}} \sum_{m \in \mathbb{Z}} c_{m}^{+}(a) \frac{e^{2 \pi i m x}}{m^{n}} .
$$

\section{NeW RESUlts ON BERNOULli AND EULER POLYNOMiAls OF HIGHER ORDER}

In this section, we give two applications of our results. We obtain new explicit formulas and Fourier series for Bernoulli and Euler polynomials of higher order.

\subsection{Bernoulli polynomials.}

We start with two applications, we obtain new characterizations of Bernoulli and Euler polynomials. Note that for $\varphi(0)=1, \varphi(k)=k, k \geq 1, a_{k}=E_{k}(0)$, is well-known that $E_{0}(0)=1$ and $E_{k}(0)=0$ for $0 \neq k$ even. Then we obtain

$$
f(t) e^{x t}=\sum_{k \text { even }} E_{k}(0) \frac{t^{k}}{k !} \cdot \frac{2 e^{x t}}{e^{t}+1}=\frac{2 e^{x t}}{e^{t}+1} .
$$

We get $P_{n}(x)=E_{n}(x)$. It means, in particular, that if the function $F: t \rightarrow f(t)-1$ is odd and $P_{n}(1-x)=(-1)^{n} P_{n}(x)$ then $P_{n}(x)=E_{n}(x)$. Similarly, one can apply it to Bernoulli polynomials $B_{n}(x)$.

We have the following general formulation. 
Theorem 3.1 (Bernoulli polynomials by symmetry). Let $\left(P_{n}(x)\right)_{n}$ be a sequence of Bernoulli's polynomials type (1.1) such that

$$
P_{n}(1-x)=(-1)^{n} P_{n}(x)
$$

Let $N$ be a positive integer. If the function $F: t \rightarrow f(t)-\sum_{k=0}^{N} B_{k}(0) \frac{t^{k}}{k !}$ is even, then we obtain

$$
P_{n}(x)=B_{n}(x), \text { and } f(t)=\frac{t}{e^{t}-1} .
$$

Proof. We apply Theorem 2.5 and equation (2.6), with $a=1, a_{k}=B_{k}(0)$. It is well-known that $B_{1}(0)=-1 / 2$ and $B_{k}(0)=0$ for $0 \neq k$ odd. Then we obtain

$$
f(t) e^{x t}=-2 \sum_{k \text { odd }} B_{k}(0) \frac{t^{k}}{k !} \cdot \frac{t e^{x t}}{e^{t}-1}=\frac{t e^{x t}}{e^{t}-1} .
$$

It means, in particular, that the function $F: t \rightarrow f(t)+\frac{t}{2}$ is even and $P_{n}(1-x)=(-1)^{n} P_{n}(x)$.

Thus we get $P_{n}(x)=B_{n}(x)$. Then we deduce the theorem.

\subsection{Euler polynomials.}

Theorem 3.2 (Euler polynomials by symmetry). Let $\left(P_{n}(x)\right)_{n}$ be a sequence of Euler's polynomials type (1.1) such that

$$
P_{n}(1-x)=(-1)^{n} P_{n}(x)
$$

Let $N$ be a positive integer. If the function $F: t \rightarrow f(t)-\sum_{k=0}^{N} E_{k}(0) \frac{t^{k}}{k !}$ is odd, then we obtain

$$
P_{n}(x)=E_{n}(x), \text { and } f(t)=\frac{2}{e^{t}+1} .
$$

Proof. Again we apply Theorem 2.5 and equation (2.5), with $a=1, a_{k}=E_{k}(0)$. It is well-known that $E_{0}(0)=1$ and $E_{k}(0)=0$ for $0 \neq k$ even. Then we obtain

$$
f(t) e^{x t}=\sum_{k \text { even }} E_{k}(0) \frac{t^{k}}{k !} \cdot \frac{2 e^{x t}}{e^{t}+1}=\frac{2 e^{x t}}{e^{t}+1} .
$$

It means, in particular, that the function $F: t \rightarrow f(t)-1$ is odd and $P_{n}(1-x)=(-1)^{n} P_{n}(x)$.

Thus we get $P_{n}(x)=E_{n}(x)$. Then we complete the proof of the theorem.

3.3. Bernoulli polynomials of order $r$. Let $r$ be a positive integer. The Bernoulli polynomials and numbers of order $r$ are given through the equations

$$
\sum_{n \geq 0} B_{n}^{(r)}(x) \frac{t^{n}}{n !}=\left(\frac{t}{e^{t}-1}\right)^{r} e^{x t}
$$


and $B_{n}^{(r)}=B_{n}^{(r)}(0)$.

The function $f(t)=\left(\frac{t / r}{e^{t / r}-1}\right)^{r}, r \geq 1$ satisfy $f(t) e^{t}=f(-t)$ and the function

$$
F(t)=f(t)-\sum_{k \geq 0} r^{-2 k-1} B_{2 k+1}^{(r)} \frac{t^{2 k+1}}{(2 k+1) !}
$$

is even function. So that we obtain

$$
r^{-n} B_{n}^{(r)}(r x)=-2 \sum_{0 \leq k \leq n / 2} r^{-2 k-1} \frac{B_{2 k+1}^{(r)}}{2 k+1}\left(\begin{array}{c}
n \\
2 k
\end{array}\right) B_{n-2 k}(x) .
$$

We have a new formula for the generalized Bernoulli polynomials $B_{n}^{(r)}(x)$

$$
B_{n}^{(r)}(x)=-2 \sum_{0 \leq k \leq n / 2} r^{n-2 k-1} \frac{B_{2 k+1}^{(r)}}{2 k+1}\left(\begin{array}{c}
n \\
2 k
\end{array}\right) B_{n-2 k}(x / r)
$$

and, thanks to the relations in [2, (1.10), Corollary 1.8], the coefficients $B_{2 k+1}^{(r)}$ are given by the formula

$$
B_{n}^{(r)}= \begin{cases}n\left(\begin{array}{c}
n-1 \\
r-1
\end{array}\right) \sum_{k=1}^{r}(-1)^{k-1} s(r, k) \frac{B_{n-r+k}}{n-r+k}, & n \geq r \\
\frac{1}{\left(\begin{array}{c}
r-1 \\
n
\end{array}\right)} s(r, r-n), & 0 \leq n \leq r-1 .\end{cases}
$$

where $s(n, l)$ is the Stirling number of the first kind.

Therefore we obtain the formulas

Theorem 3.3. For $n \geq r \geq 2$, we have

$$
\begin{array}{r}
B_{n}^{(r)}(x)=-2 r^{n-1} \sum_{0 \leq k \leq r / 2-1} r^{-2 k} \frac{s(r, r-2 k-1)}{2 k+1} \frac{\left(\begin{array}{c}
n \\
2 k
\end{array}\right)}{\left(\begin{array}{c}
r-1 \\
2 k+1
\end{array}\right)} B_{n-2 k}(x / r) \\
-2 r^{n-1} \sum_{r / 2 \leq k \leq n / 2} r^{-2 k} \frac{B_{2 k+1}^{(r)}}{2 k+1}\left(\begin{array}{c}
n \\
2 k
\end{array}\right) B_{n-2 k}(x / r),
\end{array}
$$

with

$$
\frac{B_{2 k+1}^{(r)}}{2 k+1}=\left(\begin{array}{c}
2 k \\
r-1
\end{array}\right) \sum_{j=1}^{r}(-1)^{j-1} s(r, j) \frac{B_{2 k+1-r+j}}{2 k+1-r+j}, j \geq r / 2 .
$$

We give details for $r=2,3$ which give us new explicit formulas for $B_{n}^{(2)}(x)$ and $B_{n}^{(3)}(x)$. Thanks to $B_{1}^{(2)}=s(2,1)=-1, B_{2 k+1}^{(2)}=-(2 k+1) B_{2 k},(k \geq 1)$, then we have

$$
\begin{aligned}
& B_{n}^{(2)}(x)=\sum_{0 \leq k \leq n / 2} 2^{n-2 k}\left(\begin{array}{c}
n \\
2 k
\end{array}\right) B_{2 k} B_{n-2 k}(x / 2), \\
& B_{n}^{(3)}(x)=3^{n} B_{n}(x / 3)-2 \sum_{1 \leq k \leq n / 2} 3^{n-2 k-1} \frac{B_{2 k+1}^{(3)}}{2 k+1}\left(\begin{array}{c}
n \\
2 k
\end{array}\right) B_{n-2 k}(x / 3)
\end{aligned}
$$


and then for $n \geq 4$ we have

$$
B_{n}^{(3)}(x)=3^{n} B_{n}(x / 3)+\frac{1}{2} 3^{n-2}\left(\begin{array}{l}
n \\
2
\end{array}\right) B_{n-2}(x / 3)-2 \sum_{2 \leq k \leq n / 2} 3^{n-2 k}(2 k-1)\left(\begin{array}{c}
n \\
2 k
\end{array}\right) B_{2 k} B_{n-2 k}(x / 3) .
$$

3.4. Euler polynomials of order $r$. Let $r$ be a positive integer. The Euler polynomials and numbers of order $r$ are given by the equations

$$
\sum_{n \geq 0} E_{n}^{(r)}(x) \frac{t^{n}}{n !}=\left(\frac{2}{e^{t}+1}\right)^{r} e^{x t}
$$

and $E_{n}^{r}=E_{n}^{(r)}(0)$.

Let $f(t)=\left(\frac{2}{e^{t / r}+1}\right)^{r}$. The function $f$ satisfy $f(t) e^{t}=f(-t)$ and the function

$$
F(t)=f(t)-\sum_{k \geq 0} r^{-2 k} E_{2 k}^{(r)} \frac{t^{2 k}}{(2 k) !}
$$

is odd function. So that we obtain

$$
r^{-n} E_{n}^{(r)}(r x)=\sum_{0 \leq k \leq n / 2} r^{-2 k} E_{2 k}^{(r)}\left(\begin{array}{c}
n \\
2 k
\end{array}\right) E_{n-2 k}(x)
$$

Then we get a new formula $E_{n}^{(r)}(x)$

$$
E_{n}^{(r)}(x)=\sum_{0 \leq k \leq n / 2} r^{n-2 k}\left(\begin{array}{c}
n \\
2 k
\end{array}\right) E_{2 k}^{(r)} E_{n-2 k}(x / r)
$$

On the other hand, it is well-known that the numbers $E_{n}^{(r)}$ can be express explicitly in terms of Stirling numbers of first kind and Euler numbers $E_{k}:=E_{k}(0)$. Precisely, we have

Lemma 3.1. Let $r$ be a positive integer. We have

$$
E_{n}^{(r)}=\frac{2^{r-1}}{(r-1) !} \sum_{j=0}^{r-1}(-1)^{j} s(r, r-j) E_{n+r-j-1}
$$

Hence we get the result

Theorem 3.4. Let $r$ be a positive integer. We have

$$
E_{n}^{(r)}(x)=\frac{2^{r-1}}{(r-1) !} \sum_{\substack{0 \leq j \leq r-1 \\
0 \leq k \leq n / 2}}(-1)^{j} s(r, r-j)\left(\begin{array}{c}
n \\
2 k
\end{array}\right) r^{n-2 k} E_{2 k+r-j-1} E_{n-2 k}(x / r) .
$$

The relation is obvious for $r=1$. For $r=2$ and $n \geq 2$ we obtain

$$
E_{n}^{(r)}(x)=2^{n} E_{n}(x / 2)+\sum_{1 \leq k \leq n / 2}\left(\begin{array}{c}
n \\
2 k
\end{array}\right) 2^{n+1-2 k} E_{2 k+1} E_{n-2 k}(x / r) .
$$


3.5. Fourier expansions for higher Bernoulli-Euler's polynomials. We apply our main result to get Fourier series for Euler's and Bernoulli's polynomials of higher order $r$. From our Theorem 3.3 and Theorem 3.4 we can obtain the Fourier expansions for the polynomials $B_{n}^{(r)}(x)$ and $E_{n}^{(r)}(x)$.

Theorem 3.5 (Fourier expansion). For $x \in(0, r)$ we have Fourier expansion for the Euler polynomials of order $r \geq 1$ given by

$$
E_{n}^{(r)}(x)=\frac{2^{r}}{(r-1) !} \frac{n !}{(2 \pi i)^{n+1}} \sum_{m \in \mathbb{Z}} c_{m}(n, r) \frac{e^{2 \pi i(m-1 / 2) \frac{x}{r}}}{(m-1 / 2)^{n+1}},
$$

where

$$
c_{m}(n, r)=\sum_{\substack{0 \leq j \leq r-1 \\ 0 \leq k \leq n / 2}}(-1)^{j} s(r, r-j)(\pi i)^{2 k}(2 m-1)^{2 k} E_{2 k+r-j-1} .
$$

For example for

(1) $r=1$ we have $c_{m}(n, 1)=1$ for any $m \in \mathbb{Z}$. We recover the known result about periodized Euler functions.

(2) $r=2$,

$$
c_{m}(n, 2)=1 / 2+\sum_{1 \leq k \leq n / 2}(\pi i)^{2 k}(2 m-1)^{2 k} E_{2 k+1}, n \geq 2
$$

\section{REFERENCES}

1. P. E. Appell, Sur une classe de polynômes, Annales d'école normale supérieure, s.2, v. 9 (1882) 119-144.

2. A. Bayad, M. Beck Relations for Bernoulli-Barnes numbers and Barnes zeta functions, Int. J. Number Theory 10 (2014), no. 5, pp. 1321-1335.

3. J. Bernoulli, Ars conjectandi, Basel 1713, p. 97. Posthumously published. Bernoulli died in 1705.

4. L. Euler, Methodus generalis summandi progressiones, Comment. acad. sci. Petrop., v.6 (1738), 68-97.

5. A. Hurwitz, Personal communication via George Polya that Hurwitz used the Fourier series approach to Bernoulli polynomials in his lectures.

6. S. Ikeda, On supplementary formulas for Sheffer sequences, Aequationes Math. Vol 89 (2015), Issue 4, pp.11231131.

7. D. H. Lehmer, A New Approach to Bernoulli Polynomials, The American Mathematical Monthly, v.95 (1998), no. 10, pp. 905-911.

8. E. Lucas, Théorie des Nombres, Paris 1891, Chapter 14.

9. J. L. Raabe, Zurückführung einiger Summen and bestimmten Integrale auf die Jacob Bernoullische Function, Journal für die reine and angrew. Math. 42 (1851), 348-376, especially p. 356.

10. J-P. Serre, Cours d'arithmétique, PUF, 1960.

Abdelmejid Bayad, Département de mathématiques, Université d'Evry Val d'Essonne, 23 Bd. De France, 91037 Evry Cedex, France,

E-mail address: abayad@maths.univ-evry.fr

Takao Komatsu, School of Mathematics and Statistics, Wuhan University, Wuhan, 430072, CHINA,

E-mail address: komatsu@whu.edu.cn 